\title{
CARDIAC OPERATIONS IN PATIENTS WITH LOW-GRADE SMALL LYMPHOCYTIC MALIGNANCIES
}

Probal Ghosh, FRCSEd, FACC, FETCS

Ian Carroll, $\mathrm{BSc}^{\mathrm{a}}$

Apoorva Kanhere, $\mathrm{MCh}^{\mathrm{a}}$

Bernice Ng, FRACP, FFICANZCA ${ }^{b}$

Rober Larbalestier, FRACS ${ }^{\text {a }}$

Mark Edwards, FRCSEd, FRACS ${ }^{\text {a }}$
Background: Low-grade small lymphocytic (B cell) malignancies (encompassing chronic lymphocytic leukemia and some types of non-Hodgkin lymphoma) are diseases of the elderly. Open cardiac procedures are known to have increased risk of postoperative infection and other morbidities in these immunodeficient patients. Outcome of open cardiac procedures in these patients was reviewed retrospectively. Patients: Thirteen patients (aged 58-82 years, 11 men, 2 women) with these lymphocytopathologic diseases (8 with chronic lymphocytic leukemia and 5 with non-Hodgkin lymphoma) underwent cardiac operations between January 1977 and June 1998. Mean age was $72 \pm 2.1$ years. Isolated coronary artery bypass grafting was performed in 11 and combined procedures and double valve replacement were performed in 1 each. Preoperatively, 9 patients were in a low-risk clinical stage. Mean preoperative duration of lymphocytopathologic disease was $6.1 \pm 1.6$ years. Mean preoperative New York Heart Association functional class was 2.8. Results: There was no operative death. Average stay in the intensive care unit was $41.4 \pm 8.6$ hours. Postoperative leg and superficial sternal wound infections were encountered in 3 patients. Average postoperative hospital stay was $10.0 \pm 1.7$ days. During the follow-up up to 72 months, 1 patient underwent a second cardiac operation. There was 1 late death 4 years later. Coronary stenting was done in 1 patient and a cardioverter-defibrillator was implanted in another patient for recurrent angina. Three patients underwent chemotherapy. Cardiac and lymphocytopathologic status remained stable in others. Conclusions: Acceptable outcome may be anticipated after cardiac operations in patients with low-grade chronic lymphocytic leukemia and non-Hodgkin lymphoma in early stages. However, the possibility of infection and progression of cardiac and lymphocytopathologic status in these patients should call for caution. (J Thorac Cardiovasc Surg 1999;118:1033-7)
A ging of the cardiac surgical population in most adult cardiac surgical centers in recent times has focused attention on associated comorbidities, possible complications, and expected outcomes in the elderly population. Lymphocytic malignant disease is a comorbidity in this subset of aged patients.

Most small lymphocytic malignancies are of B-cell

From the Departments of Cardiothoracic Surgerya and Intensive Care Medicine, ${ }^{\mathrm{b}}$ Royal Perth Hospital, Perth, West Australia.

Received for publication Dec 1, 1998; revisions requested Jan 29, 1999; revisions received July 22, 1999; accepted for publication Sept 7, 1999.

Address for reprints: Professor Probal Ghosh, Harav Zinger 8, Rishon Le Zion 75255, Israel (E-mail: probalg@ @otmail.com).

Copyright $(\odot) 1999$ by Mosby, Inc.

0022-5223/99 $\$ 8.00+0 \quad \mathbf{1 2 / 1 / 1 0 2 8 1 7}$ type. These diseases involve blood, bone marrow, lymph nodes, and other organs. Three morphologic subtypes of small lymphocytic malignancies of B-cell type have been recognized: (1) chronic lymphocytic leukemia (CLL), (2) small lymphocytic malignant lymphoma, and (3) small lymphocytic-plasmacytoid malignant lymphoma. Presence or absence of blood involvement and plasmacytoid differentiation distinguish these subtypes. ${ }^{1}$

Rai and colleagues ${ }^{2}$ classified CLL in 0 to IV clinical stages: stage 0, lymphocytosis in blood and marrow only; stage I, lymphocytosis associated with enlarged nodes; stage II, lymphocytosis with splenomegaly or/and hepatomegaly; stage III, lymphocytosis with anemia $(<110 \mathrm{~g} / \mathrm{L})$; and stage IV, lymphocytosis with thrombocytopenia ( $<100$ platelets $\left./ \mathrm{mm}^{3}\right)$. Lymphadenopathy may or may not be present in stages II to IV. 
Splenomegaly and hepatomegaly are not essential features in stages III and IV.

For expedient clinical decision making, the Rai staging system of CLL has been modified into 3 groups by estimating the disease severity and prognosis: low-, intermediate-, and high-risk groups. Rai stage 0 is considered to indicate a low-risk group, in which the duration of survival is similar to that of the "normal" population and therapy is not indicated. The intermediate-risk group comprises Rai stages I and II, and the high-risk group comprises Rai stages III and IV. These risk groups are similar to three stages $\mathrm{A}, \mathrm{B}$, and $\mathrm{C}$ of the Binet system ${ }^{3}$ and signify similar prognoses.

Similarly the classification of non-Hodgkin lymphoma (NHL) has evolved through several changes into a clinically relevant Working Formulation widely used in the United States. In the Working Formulation, the lymphomas are categorized again as low grade, intermediate grade, and high grade, indicating aggressiveness of the neoplasm.

The natural history after treatment of Rai stage 0 or modified Rai low-risk or Binet stage A of CLL and low-grade NHL in the Working Formulation appears to have similar estimated survival and similar biologic behavior. Recently the International Lymphoma Study Group proposed a more comprehensive and complex REAL (Revised European-American Lymphoma) classification covering NHL, lymphoid leukemias, plasma cell neoplasms, and Hodgkin disease. ${ }^{4}$ It takes into consideration morphologic and functional properties of the neoplastic cells and the stage of maturation of the lineage cell (B or T cell). Both CLL and low-grade NHL appear to originate from the stage of B-cell pre-antigen exposure.

Leukemia has been anecdotally observed as a comorbidity in elderly patients requiring cardiac surgery. ${ }^{5-7}$ Myocardial ischemia caused by external compression of the left main coronary artery by mediastinal lymphoma has been reported. ${ }^{8}$ NHL directly involving the heart has been reported only twice in the surgical literature. ${ }^{9,10}$ There are very few focused surgical reports ${ }^{11-13}$ of cardiac operations in patients with CLL. All of them indicated a possible increase in postoperative infection rate. From this perspective, we reviewed the Royal Perth Hospital experience of cardiac operations in this subset of patients.

\section{Patients and methods}

From January 1977 through June 1998, 13 patients with documented B-cell small lymphocytic malignancies underwent cardiac operations.

CLL was diagnosed before the operation in 8 patients. CLL was diagnosed by the minimum criterion of lymphocytosis in peripheral blood $\left(>5 \times 10^{9} / \mathrm{L}\right)$. The diagnosis was a preoperative surprise on routine hematologic work-up in 2 of them. The remaining 5 patients, preoperatively having a diagnosis of small lymphocytic malignant lymphoma, were considered to have NHL.

The status of all patients was also categorized into low-risk (A), intermediate-risk (B), and high-risk (C) clinical stages.

Clinical records of all patients were retrospectively analyzed. Current follow-up was conducted by telephone with their general practitioners about their recent cardiac and lymphocytopathologic status. All variables were expressed as mean \pm standard error.

\section{Results}

The mean age of the 13 patients was $72.0 \pm 2.1$ years. They represented $0.1 \%$ of all patients undergoing coronary artery bypass grafting (CABG), $0.07 \%$ of all patients having valve procedures, and $0.08 \%$ of all patients having cardiac operations. Ten patients were more than 65 years old and represented $0.63 \%$ of all patients undergoing cardiac surgery who were older than 65 years.

Table I details the clinical data. Although the hematologic diagnosis was a preoperative surprise in 2 patients, the average preoperative duration of lymphocytopathologic disease was $6.1 \pm 1.6$ years. Twelve of the patients were in low-risk clinical stage A. All patients with NHL had low-grade lymphomas (Working Formulation) with small cleaved cells. Only 1 patient (with NHL) had received preoperative chemotherapy with chlorambucil. Preoperative anemia was present in 4 patients with CLL and 1 patient with NHL.

Isolated $\mathrm{CABG}$ was performed in 11 patients, $\mathrm{CABG}$ with mitral valve replacement (MVR) in 1, and MVR with aortic valve replacement (AVR) in 1 patient. An average $3.1 \pm 1.2$ vessels were grafted. The left internal thoracic artery was used as a conduit in 8 patients.

Table II details the perioperative data. Stay in the intensive care unit was prolonged in 3 patients with CLL (72 hours, 96 hours, and 96 hours) and in 1 patient with NHL (72 hours); 2 of them had undergone emergency CABG. One patient had required intra-aortic balloon pumping. Perioperative myocardial infarction with a significant rise of cardiac enzymes was noticed in only these 2 patients.

All patients received routine prophylaxis in conformity with the antibiotic regimen of the period. During this decade various antibiotic regimens (floxacillin [INN: flucloxacillin] with gentamicin/ceftriaxone/vancomycin) have been used in this unit. Postoperative superficial sternal wound infection was noticed in 1 patient with NHL and leg wound infections in 2 
Table I. Clinical data

\begin{tabular}{lccc}
\hline & $C L L$ & $N H L$ & Total \\
\hline No. & 8 & 5 & 13 \\
Sex (male/female) & $7 / 1$ & $4 / 1$ & $11 / 2$ \\
Age (y) & & & $72.0 \pm 2.1$ \\
$\quad$ Mean & $76.0 \pm 2.1$ & $66.0 \pm 2.5$ & $58-82$ \\
$\quad$ Range & $64-82$ & $58-71$ & 12 \\
Preop clinical stage & & 5 & 1 \\
$\quad$ A & 7 & 0 & 6.1 \\
B & 1 & 5.75 & 2.8 \\
Preop mean duration of CLL/NHL (y) & 6.5 & 2.6 & $14.5 \pm 5.3$ \\
Preop mean NYHA & 2.9 & $14.8 \pm 2.4$ & $25.5 \pm 3.2$ \\
Preop Hgb (g/dL) & $11.3 \pm 1.6$ & $22.0 \pm 4.7$ & $7.4 \pm 3.5$ \\
Preop globulin (g/dL) & $30.0 \pm 2.8$ & $3.2 \pm 0.4$ & \\
Preop TLC (k/mL) & $32.1 \pm 16.0$ & $206.4 \pm 35.1$ & $192.95 \pm 40.3$ \\
Preop lymphocytes (k/mL) & $26.6 \pm 15.6$ & 1 & 5 \\
Preop platelets (k/mL) & $179.0 \pm 42.5$ & & \\
Preop LV dysfunction & 4 & & \\
\hline
\end{tabular}

Preop, Preoperative; $C L L$, chronic lymphocytic leukemia; NHL, non-Hodgkin lymphoma; NYHA, New York Heart Association; $H g b$, hemoglobin; $T L C$, total leukocyte count; $L V$, left ventricle.

Table II. Perioperative data

\begin{tabular}{lccc}
\hline & $C L L$ & $N H L$ & Total \\
\hline Operation & 3 & 4 & 7 \\
$\quad$ Elective & 4 & 0 & 4 \\
Urgent & 1 & 1 & 2 \\
Emergency & $103.9 \pm 17.8$ & $70.3 \pm 13.3$ & $92.6 \pm 13.2$ \\
CPB time (min) & $59.7 \pm 11.4$ & $40.2 \pm 10.7$ & $61.0 \pm 9.0$ \\
Crossclamp time (min) & & & \\
Units of blood/products given per & 2.6 & 1.6 & 2.2 \\
patient & 0.9 & 1.6 & 1.1 \\
Packed RBC (avg) & 3.4 & 0.9 & 2.6 \\
Fresh frozen plasma (avg) & $49.5 \pm 12.7$ & $39.0 \pm 11.3$ & $43.4 \pm 8.6$ \\
Platelets (avg) & $11.2 \pm 2.2$ & $7.6 \pm 0.8$ & $10.0 \pm 1.7$ \\
ICU stay (h) & & & $7-76$ \\
Postop hospital stay (d) & $7-76$ & $8-23$ & $30.3 \pm 6.4$ \\
Follow-up duration (mo) & $36.3 \pm 9.4$ & $20.6 \pm 5.7$ & 9 \\
Range & & & 2 \\
Mean & 5 & 4 & 1 \\
Current CLL/NHL stage & 1 & 1 &
\end{tabular}

$\overline{C L L}$, Chronic lymphocytic leukemia; $N H L$, non-Hodgkin lymphoma; $C P B$, cardiopulmonary bypass; $R B C$, red blood cell; avg, average; $I C U$, intensive care unit; Postop, postoperative.

patients with CLL, but their postoperative hospital stays were not prolonged. The causative microbes were Klebsiella and Pseudomonas. One patient with CLL undergoing MVR and AVR had a blood culture growing Pseudomonas aeruginosa and was treated with intravenous antibiotics for 6 weeks. The same patient had required postoperative intra-aortic balloon pumping. Average postoperative hospital stay was $10.0 \pm 1.7$ days. It was extended to 14 days because of postopera- tive atrial fibrillation in only 1 patient with CLL who underwent urgent $\mathrm{CABG}(\times 5)$ for unstable angina.

There was no hospital death. Follow-up was available for all patients. There was 1 late death 4 years after MVR and CABG in a patient with CLL. He had undergone AVR and coronary stenting 3 years after his first operation. Angina recurred in 2 other patients: One patient (CLL) underwent stenting of the right coronary artery 3 years after $C A B G \times 5$. The second patient 
Table III. Literature review of cardiac surgery in CLL

\begin{tabular}{|c|c|c|c|c|c|}
\hline First author & Place & Year & No & Age (y) & $\operatorname{Sex}$ \\
\hline Galvin $^{6}$ & United Kingdom & 1988 & 1 & $>70$ & \\
\hline Paccagnella $^{12}$ & Italy & 1988 & 1 & 62 & M \\
\hline Fox $^{11}$ & United States & 1988 & 2 & 62,69 & M \\
\hline Finck $^{13}$ & United States & 1993 & 26 & $60-79(69.6 \pm 4.9)$ & $19 \mathrm{M}, 7 \mathrm{~F}$ \\
\hline Ghosh & Australia & 1999 & 8 & $64-82$ & $7 \mathrm{M}, 1 \mathrm{~F}$ \\
\hline
\end{tabular}

Preop, Preoperative; $A V R$, aortic valve replacement; chemo, chemotherapy; $P O D$, postoperative day; $M$, male; $F$, female; $C A B G$, coronary artery bypass grafting; $M V R$, mitral valve replacement.

(NHL) had implantation of a cardioverter-defibrillator 1 year after primary CABG for inducible ventricular tachycardia on electrophysiologic studies.

Three patients required chemotherapy during the follow-up period. One of them had pancytopenia develop after cyclophosphamide therapy after progression of CLL to stage IV and died of profound sepsis and obstructive jaundice caused by extrahepatic cholestasis. Another patient with CLL received a course of chlorambucil 3 years after the operation because of progression of CLL. The third patient (with NHL) required 3 cycles of chemotherapy (cyclophosphamide, doxorubicin [Adriamycin\}, vincristine, and prednisolone) with subsequent remission. Lymphocytopathologic status remained stable in the remaining 10 patients ( 4 with NHL and 6 with CLL).

\section{Discussion}

Lymphocytic malignant diseases are afflictions of elderly patients, the median age at diagnosis being above 61 years. Occurrence in patients below 30 years of age is rare. In CLL $90 \%$ of patients are more than 50 years of age and $70 \%$ are more than 60 years old. ${ }^{14}$ Rai and associates ${ }^{2}$ estimated that although some patients with CLL may die within 1 year after diagnosis, a large number of them will live longer than 10 years.

Monoclonal expansion of B cells is etiogenic of CLL in $95 \%$ of cases. Only 5\% of CLLs arise from T cells, usually with skin involvement. Immune deficiency states are known to occur with some of these B-cell lymphopathies. The natural history of CLL has been changing during the past 3 decades. Currently, the patients are much older and a large proportion of them are in the low-risk Binet A clinical stage. ${ }^{15}$ Estimated survivals are as follows: stage A, 10 to 12.5 years; stage $\mathrm{B}, 7$ to 8 years; and stage C, 2.5 to 3.5 years. ${ }^{16,17}$

Low-grade NHLs are slow-growing indolent neoplasms with estimated median survivals of 8 to 10 years untreated. They are responsive to chemotherapy, but treatment does not prolong survival. They can transform into high-grade lymphomas and behave aggressively.
All but 1 of our patients with CLL were in a low-risk group at the time of operation; 2 of them progressed to stage B and C during the follow-up. Similarly, all but 2 patients with NHL continued to have low-grade disease during the follow-up. Mean age at operation was 72.0 \pm 2.1 years. The natural life expectancy in Western Australia is 75 years for men and 80.7 years for women. Thus significant attrition of life expectancy has not been noted in this subset of patients with low-grade B-cell malignant disease. Lack of operative mortality in this reported group compares favorably with that of the general cardiac surgical population.

Postoperative pneumonia has been noted in $4(13 \%)$ of 30 previously reported cases of CLL (Table III). It was not observed in the present series, although postoperative atelectasis was noticed in 2 patients. Postoperative chest or leg wound infection was noticed in 3 patients, an incidence that was not remarkably different from that observed in other patients undergoing cardiac operations. Neither intensive care unit stay nor postoperative hospital stay nor perioperative morbidity was longer in this group of patients than in patients having general cardiac surgery. Systemic inflammatory response and immunologic changes after routine cardiopulmonary bypass have been studied in the past.

The immune response pattern has also been noted to be altered in elderly patients. The extent of such subclinical immune changes in these immunocompromised patients after cardiac surgery has not been reported in the literature. Progression of cardiac disease in 3 patients during follow-up reflected the usual natural history and is possibly uninfluenced by the comorbidity.

CLL has been observed to be a cause of late death. ${ }^{7}$ Progress of CLL from intermediate-risk to high-risk stage possibly contributed to the only late death in this series in a 79-year-old patient.

Progression of NHL in 1 patient could be reversed with chemotherapy during follow-up. No specific preoperative variable could be identified in these patients to prognosticate perioperative infection or progression 


\begin{tabular}{lll}
\hline Operation & Preop treatment & Outcome \\
\hline AVR & & \\
AVR & Preop chemo, steroid & Postop pneumonia, POD 5 \\
CABG & Preop chemo in 1 & Uneventful \\
CABG (+assoc operation in 7) & & Pneumonia in 3; death in 2 \\
CABG in 6; CABG + MVR in 1; AVR + MVR in 1 & & Leg wound infection in 2 \\
\hline
\end{tabular}

of lymphocytopathologic disease or cardiac status. However, Finck and associates ${ }^{13}$ had observed "significant difference in absolute neutrophil count" between their 6 patients in whom postoperative infection developed $\left(4.3 \pm 2.1 \times 10^{9} / \mathrm{L}\right)$ and 20 patients in whom infection did not develop $\left(6.1 \pm 3.4 \times 10^{9} / \mathrm{L}\right)$.

In conclusion, the expected outcome after cardiac surgery in patients with low-grade B-cell malignant disease parallels the result in the similar elderly age groups. However, the possible risk of postoperative infection, progression of cardiac disease, and lymphoproliferative state should call for caution.

\section{REFERENCES}

1. Dick FR. Chronic lymphocytic leukemia and related small lymphocytic malignancies. In: Jaffe ES, editor. Major problems in pathology (MPP 16): surgical pathology of lymph node and related organs. 2nd ed. Philadelphia: WB Saunders; 1995. p. 205-20.

2. Rai KR, Sawitsky A, Cronkite EP, Chanana AD, Levy RN, Pasternack BS. Clinical staging of chronic lymphocytic leukemia. Blood 1975;46:219-34.

3. Binet JL, Catovsky D, Dighiero G, et al. Chronic lymphocytic leukemia: recommendations for diagnosis, staging and response criteria. Ann Intern Med 1989;110:236-8.

4. Harris NL, Jaffe ES, Stein H, et al. A revised European-American classification of lymphoid neoplasms: a proposal from International Study Group. Blood 1994;87:1361.

5. Glock Y, Pecoul R, Cerene A, Laguere J, Puel P. Aortic valve replacement in elderly patients. J Cardiovasc Surg 1984;25:205-10.

6. Galvin I, Mosieri J, Paneth M, Gibson D. An analysis of isolated aortic valve surgery and combined procedures in patients over 70 years of age. J Cardiovasc Surg 1988;29:577-81.

7. Taniyasu N, Koh YS, Yokoyama S, Takenaka A, Ikawa O. Coronary artery bypass grafting in an octogenarian with chronic myelomonocytic leukemia. Nippon Kyobu Geka Gakkai Zashi 1997;45:750-3.

8. Zeymer U, Hirschmann WD, Neuhaus KL. Left main coronary stenosis by a mediastinal lymphoma. Clin Invest 1992;70:1024-6.

9. Gill PS, Chandraratna PAN, Meyer PR. Malignant lymphoma: cardiac involvement at initial presentation. J Clin Oncol 1987;5:21-4.

10. Horowitz MD, Cox MM, Neibart RM, Blaker AM, Interian A Jr. Resection of right atrial lymphoma in a patient with AIDS. Int J Cardiol 1992;34:139-42.

11. Fox LS, Schooley WR, Nichols RL, Webb WR. Open cardiac operations in patients with abnormalities of white blood cell number or function. South Med J 1988;81:1065-6.

12. Paccagnella A, De Angeli S, Caenaro G, Zussa C, Valfré C, Simini G. Cardiopulmonary bypass in leukemic patients [letter]. Ann Thorac Surg 1988;45:588.

13. Finck SJ, Cockerill KJ, Jeter JE, Orszulak TA. Coronary artery bypass grafting in patients with chronic lymphocytic leukemia. Ann Thorac Surg 1993;55:1192-6.

14. Johnson LE. Chronic lymphocytic leukemia. Am Fam Physician 1988;38:167-76.

15. Rozman C, Bosch F, Montserrat E. Chronic lymphocytic leukemia: A changing natural history? Leukemia 1997;11:775-8.

16. Kamiris T, Rohatiner AZ, Love S, et al. The management of chronic lymphocytic leukemia at a single center over a 24 year period: prognostic factors for survival. Hematol Oncol 1994;12: 29-39.

17. Lee GR, Bithell TC, Foerster J, Athens JW, Lukens JN. In: Wintrobe's clinical hematology. 9th ed, chap 78. Philadelphia: Lippincott; 1993. Vol 2. p. 2035-2142. 